\title{
Bedside Ultrasonography in Evaluating Mediastinum Leakage in an Extremely-Low- Birth-Weight Infant with Esophageal Perforation
}

\author{
Hiroshi Kawashima, MD 4 \\ ${ }^{1}$ Department of Neonatology, Saitama Children's Medical Center, \\ Saitama, Saitama, Japan \\ 2 Department of Pediatrics, Saitama Medical Center, Saitama Medical \\ University, Kawagoe, Saitama, Japan \\ ${ }^{3}$ Department of Radiology, Saitama Children's Medical Center, \\ Saitama, Saitama, Japan \\ ${ }^{4}$ Department of Pediatric Surgery, Saitama Children's Medical Center, \\ Saitama, Saitama, Japan \\ ${ }^{5}$ Department of Pediatric Surgery, The University of Tokyo Hospital, \\ Bunkyo-ku, Tokyo, Japan
}

Mitsuhiro Haga, MD ${ }^{1,2}$ Yumiko Sato, MD ${ }^{3}$ Tomo Kakihara, MD ${ }^{4,5}$ Wakako Sumiya, MD

Masayuki Kanno, MD ${ }^{1}$ Tetsuya Ishimaru, MD, PhD ${ }^{4}$ Masaki Shimizu, MD, $\mathrm{PhD}^{1}$

\author{
Address for correspondence Mitsuhiro Haga, MD, Department of \\ Pediatrics, Saitama Medical Center, Saitama Medical University, \\ Kamoda 1981, Kawagoe, Saitama, 350-8550, Japan \\ (e-mail: haga_m@saitama-med.ac.jp).
}

AJP Rep 2022;12:e76-e79.

\author{
Abstract \\ Keywords \\ - esophageal \\ perforation \\ - mediastinum leakage \\ - ultrasonography \\ - extremely-low-birth- \\ weight infant
}

Esophageal perforation in premature infants is a life-threatening condition that requires prompt treatment. Contrast-enhanced computed tomography (CECT) is recommended for diagnosis. However, it is difficult to obtain CECT images in premature infants because of their unstable conditions. We encountered a case of esophageal perforation in an extremely-low-birth-weight female infant. Bedside ultrasonography was useful in the diagnosis and follow-up evaluation of leakage in the mediastinum. Ultrasonography can be a useful modality for the evaluation of perforation of the lower part of the esophagus in premature infants.
Esophageal perforation is a rare but life-threatening condition in all ages of the population. ${ }^{1}$ As the esophagus lacks a serosal layer and is surrounded by loose areolar tissue, bacteria and digestive enzymes can easily spread to the mediastinum once the esophagus is perforated. ${ }^{2}$ Most neonatal cases of esophageal perforation are caused by medical procedures, such as gastric tube insertion, endotracheal intubation, and oropharynx suctioning. ${ }^{3}$ Premature neonates are much more susceptible to this injury than term infants owing to tissue friability. ${ }^{4}$ Prompt diagnosis and treatment based on accurate evaluation are indispensable to avoid fatal outcome. We encountered a case of esophageal perforation in an extremely-low-birth-weight infant (ELBWI) for whom ultrasonography was useful in the diagnosis and follow-up evaluation.

\section{Case Report}

Our patient's mother was a 28-year-old, gravida 2 para 1 woman. The pregnancy course was complicated with threatened preterm delivery due to chorioamnionitis. A female infant was vaginally delivered at 22 weeks and 5 days of received

February 1, 2021

accepted after revision

April 13, 2021
DOI https://doi.org/ $10.1055 / \mathrm{s}-0041-1741538$. ISSN 2157-6998.

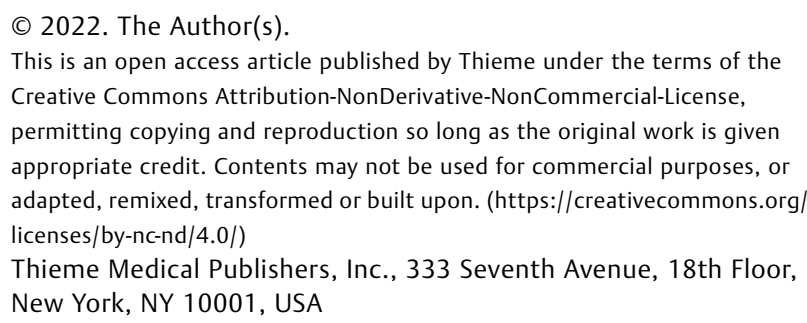


gestational age. The birth body weight was $500 \mathrm{~g}$, and Apgar scores were 2 at 1 minute and 4 at 5 minutes. She was intubated at the delivery room immediately after birth, and a gastric tube was inserted without any resistance. She was hospitalized to our neonatal intensive care unit and treated with artificial surfactant replacement for respiratory distress syndrome. She developed grade II intraventricular hemorrhage at 2 days of life.

The gastric tube was replaced at 7 days of life as a routine procedure, and its placement was confirmed with auscultation method. Further, $0.5 \mathrm{~mL}$ of the mother's milk was administered through the tube every 3 hour after replacement. On the following day (8 days of life), a small amount of blood was found in the aspirate from the gastric tube. ChestX-ray showed an oval-shaped radiolucent area in the lower part of the mediastinum and malposition of the gastric tube (-Fig. 1). Bedside ultrasonography with 6 to $15 \mathrm{MHz}$ linear transducer (LOGIQ S8; GE Healthcare, Waukesha, WI) was performed by a radiologist who had much experience in pediatric ultrasonography. It showed a round-shaped hypoechoic area under a hyperechoic line on the right anterior side of the lower thoracic esophagus (-Fig. 2A). The maximum diameter of the hypoechoic area was approximately $16 \mathrm{~mm}$ in the sagittal view (-Fig. 2B). These sonographic findings were consistent with the collection of air (hyperechoic line) and fluid (hypoechoic area) in the posterior mediastinum. With the findings of plain radiography, we diagnosed esophageal perforation due to the malposition of the gastric tube.

The gastric tube was immediately removed after the diagnosis was made. The patient was treated with total parental nutrition and broad-spectrum antibiotics and antifungal drugs. For decompressing the stomach and aspirating the digestive fluid, we carefully inserted feeding tubes in the stomach and the middle part of the esophagus while intermittently capturing X-ray photographs using a portable Xray machine.

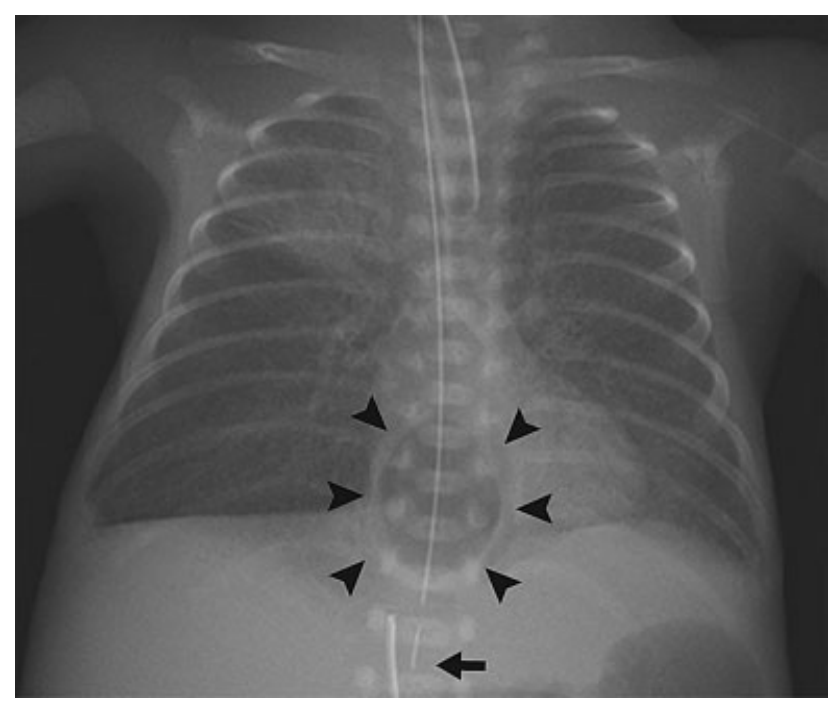

Fig. 1 The chest X-ray shows oval-shaped radiolucent area in the lower mediastinum (arrowheads). The gastric tube is descended into the radiolucent area and its distal tip is located in the middle of the upper abdomen (arrow).
The radiolucent area was not detectable on the chest X-ray photograph taken on the following day (9 days of life) (-Fig. 3). However, the hypoechoic area in the posterior mediastinum could still be observed with ultrasonography, which indicated fluid collection around the perforation site (-Fig. 4). The size of the hypoechoic area decreased gradually, and became undetectable with ultrasonography on 11 days of life. Contrast esophagography was performed on 17 days of life (10 days after perforation). There was no leakage or constriction in the esophagus. We started enteral tube feeding of the mother's milk after esophagography. The administration of antibiotics and antifungal drugs was discontinued after the course of 14 days.

The patient had acquired cytomegalovirus infection at 1 month of life and was treated with antiviral therapy. She also developed severe bronchopulmonary dysplasia requiring home oxygen therapy. However, there was no sign of recurrence of esophageal perforation or abscess formation. She was discharged at 215 days of age (53 weeks of postmenstrual age).

\section{Discussions}

To the best of our knowledge, there are only two reports describing ultrasonographic findings of esophageal perforation. ${ }^{5,6}$ Maruyama et al reported that ultrasonography was useful in detecting malposition of the gastric tube in an ELBWI with esophageal perforation. Derr and Drake reported pneumopericardium and fluid collection in the chest and abdomen of a 69-year-old man with esophageal perforation. This is the first case in which the collection of air and fluid in the posterior mediastinum was detected with ultrasonography in a patient with esophageal perforation.

Contrast-enhanced computed tomography (CECT) is the recommended imaging method in the diagnosis of esophageal perforation because of its high sensitivity and capability of eliminating other mimicking conditions. ${ }^{1}$ The typical CECT finding of esophageal perforation is the collection of air and fluid in the surrounding area of the esophagus. ${ }^{1}$ However, it is difficult to perform CECT in extremely premature infants because of the risk associated with the transfer to the CT room and intravenous contrast medium administration. Hence, plain radiography and contrast esophagography have been utilized in the diagnosis of esophageal perforation in preterm neonates. ${ }^{7}$ The important findings in plain radiography are abnormal positions of gastric tubes and air leakage. $^{7}$ Contrast esophagography is also useful in differentiating esophageal atresia from esophageal wall injury with a false lumen. ${ }^{7}$ However, both imaging methods were unable to provide information on the extent of fluid collection in the mediastinum. Further, they are not useful in detecting the development of mediastinal abscess, which is one of the most serious complications of esophageal perforation. ${ }^{8}$ Although nonoperative conservative management has consistently shown satisfactory prognosis in several case series of neonatal esophageal perforation, ${ }^{3,7,9-11}$ surgical interventions are required when the abscess is formed within the mediastinum. ${ }^{7,12,13}$ 


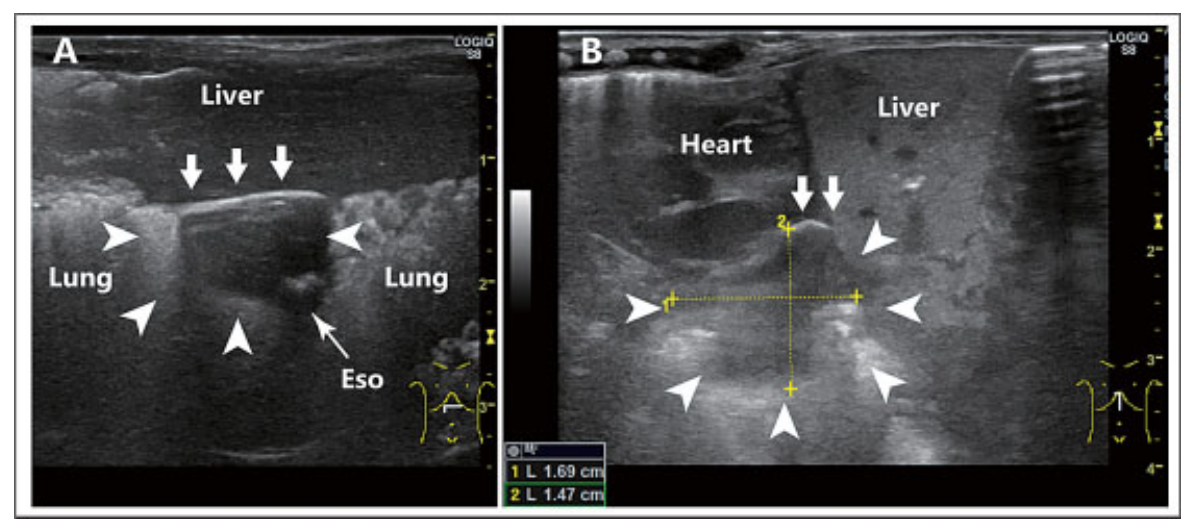

Fig. 2 Transabdominal ultrasonography shows round-shaped hypoechoic area (arrowheads) under a hyperechoic line (thick arrows) in the lower mediastinum in the axial view (A). The esophagus is on the dorsal side of the hypoechoic area (thin arrow). The maximum diameter of the echofree space was approximately $16 \mathrm{~mm}$ in the sagittal view (B).

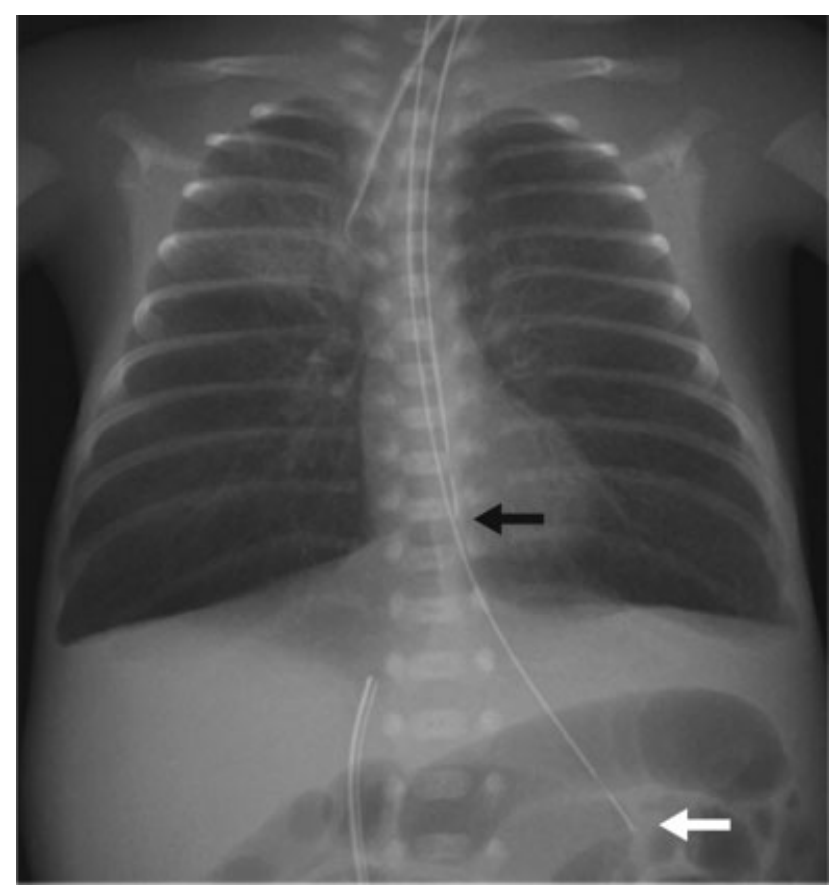

Fig. 3 No radiolucent area is observed in the mediastinum. The tubes are inserted in the stomach (white arrow) and in the middle part of the esophagus (black arrow).

Ultrasonography is the preferred imaging modality in the clinical setting of neonatal intensive care because of its low invasiveness. It can be performed at bedside without radiation exposure or contrast medium administration. Although ultrasonography cannot scan the whole mediastinum, it can be used to evaluate the lower part of the mediastinum. The technique for visualizing the abdominal and lower thoracic esophagus with ultrasonography is a well-established method that aids in the diagnosis of gastroesophageal reflux disease and hiatal hernia in infants and children. ${ }^{14,15}$ Using this technique, we were able to detect and observe the time course of the collection of air and fluid in the posterior mediastinum of the ELBWI with esophageal perforation. The

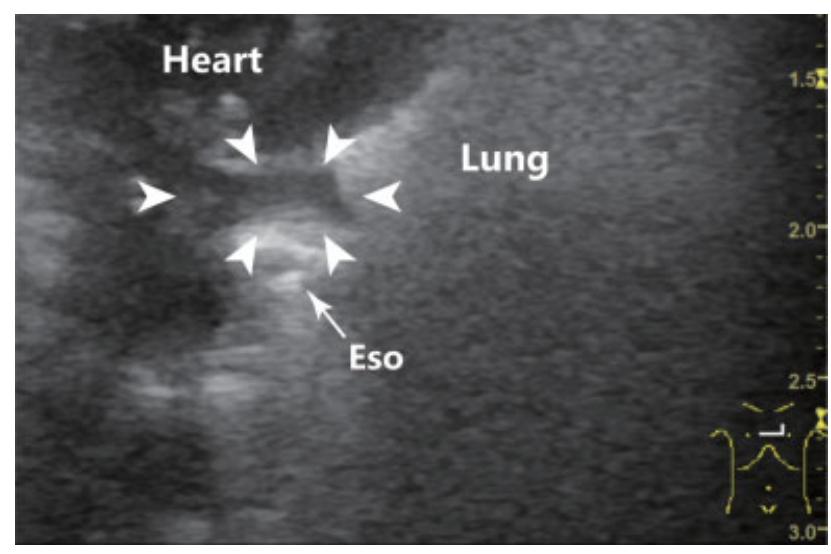

Fig. 4 Axial view of the lower mediastinum revealing reduced size of the hypoechoic area (arrowheads) in the lower mediastinum on the following day after the diagnosis of esophageal perforation (maximum diameter of the echo-free space is around $10 \mathrm{~mm}$ in axial view).

technique for visualizing the lower part of the esophagus was also practicable for nonexperts. In our case, the first evaluation was performed by an experienced radiologist, but the follow-up evaluation was performed by neonatologists. As only the lower part of the mediastinum was visualized with ultrasonography, we could not completely deny the possibility that abscess was formed in other parts of the mediastinum. However, since the size of the hypoechoic space decreased after the treatment was started, we evaluated that inflammation in the mediastinum was controlled. In fact, there was no sign of relapse of inflammation after discontinuation of the antibiotic and antifungal therapy.

$\mathrm{X}$-ray is superior to ultrasonography in finding free air and malposition of medical devices; hence, plain radiography is ideal for diagnosing esophageal perforation. However, it cannot provide much information about fluid collection in the mediastinum. Conversely, ultrasonography can detect fluid collection in the lower mediastinum. Therefore, we believe that combining plain radiography and ultrasonography is useful for diagnosing and evaluating the clinical course 
of esophageal perforation in preterm neonates, in whom performing CECT is challenging.

\section{Conflict of Interest}

The authors declare there is no conflict of interest associated with this manuscript.

\section{Acknowledgments}

The authors would like to show our appreciation to the nursing staff and radiological technologists in Saitama Children's Medical Center for their assistance for collecting the clinical data. The authors also would like to thank Enago (www.enago.jp) for the English language review.

\section{References}

1 Chirica M, Kelly MD, Siboni S, et al. Esophageal emergencies: WSES guidelines. World J Emerg Surg 2019;14:26

2 Engum SA, Grosfeld JL, West KW, Rescorla FJ, Scherer LR, Vaughan WG. Improved survival in children with esophageal perforation. Arch Surg 1996;131(06):604-610, discussion 611

3 Hesketh AJ, Behr CA, Soffer SZ, Hong AR, Glick RD. Neonatal esophageal perforation: nonoperative management. J Surg Res 2015;198(01):1-6

4 Jones KE, Wagener S, Willetts IE, Lakhoo K. Oesophageal perforation in extreme prematurity. BMJ Case Rep 2012;2012:x

5 Maruyama K, Shiojima T, Koizumi T. Sonographic detection of a malpositioned feeding tube causing esophageal perforation in a neonate. J Clin Ultrasound 2003;31(02):108-110
6 Derr C, Drake JM. Esophageal rupture diagnosed with bedside ultrasound. Am J Emerg Med 2012;30(09):2093.e1-2093.e3

7 Wolf JA, Myers EH, Remon JI, Blumfield E. Imaging findings of iatrogenic pharyngeal and esophageal injuries in neonates. Pediatr Radiol 2018;48(12):1806-1813

8 Sohda M, Kuwano H, Sakai M, et al. A national survey on esophageal perforation: study of cases at accredited institutions by the Japanese Esophagus Society. Esophagus 2020;17(03): 230-238

9 Onwuka EA, Saadai P, Boomer LA, Nwomeh BC. Nonoperative management of esophageal perforations in the newborn. J Surg Res 2016;205(01):102-107

10 Yong SB, Ma JS, Chen FS, Chung MY, Yang KD. Nasogastric tube placement and esophageal perforation in extremely low birth weight infants. Pediatr Neonatol 2016;57(05): 427-430

11 Hodgson K, Togo A, Moore AM, Moody A, King SK, Zani A. Neonatal oesophageal perforation: the role for non-operative management. J Paediatr Child Health 2018;54(08):872-874

12 Mollitt DL, Schullinger JN, Santulli TV. Selective management of iatrogenic esophageal perforation in the newborn. J Pediatr Surg 1981;16(06):989-993

13 Sapin E, Gumpert L, Bonnard A, et al. Iatrogenic pharyngoesophageal perforation in premature infants. Eur J Pediatr Surg 2000; 10(02):83-87

14 Westra SJ, Wolf BH, Staalman CR. Ultrasound diagnosis of gastroesophageal reflux and hiatal hernia in infants and young children. J Clin Ultrasound 1990;18(06):477-485

15 Savino A, Cecamore C, Matronola MF, et al. US in the diagnosis of gastroesophageal reflux in children. Pediatr Radiol 2012;42(05): $515-524$ 